\title{
Classical T Tauri stars as sources of parsec-scale optical outflows
}

\author{
F. McGroarty and T. P. Ray
}

Dublin Institute for Advanced Studies, 5 Merrion Square, Dublin 2, Ireland

Received 29 January 2004 / Accepted 26 March 2004

\begin{abstract}
Previous studies of young stellar objects (YSOs) have uncovered a number of associated parsec-scale optical outflows, the majority of which are driven by low-mass, embedded Class I sources. Here we examine more evolved Classical T Tauri stars (CTTSs), i.e. Class II sources, to determine whether these are also capable of driving parsec-scale outflows. Five such sources are presented here - CW Tau, DG Tau, DO Tau, HV Tau C and RW Aur, all of which show optical evidence for outflows of the order of $1 \mathrm{pc}$ ( $24^{\prime}$ at the distance of Taurus-Auriga). These sources were previously known only to drive "microjets" or small-scale outflows $\$ 1$ ' in length. A parsec-scale outflow from a less evolved source (DG Tau B) which was noted in the course of this work is also included here. Examination of the five newly discovered large-scale outflows from CTTSs shows that they have comparable morphologies, apparent dynamical timescales and degrees of collimation to those from less evolved sources. There is also strong evidence that these outflows have blown out of their parent molecular clouds. Finally we note that the "fossil record" provided by these outflows suggests their sources could have undergone FU Orionis-type outbursts in the past.
\end{abstract}

Key words. ISM: Herbig-Haro objects - ISM: individual objects: CW Tau - ISM: individual objects: DG Tau ISM: individual objects: DO Tau - ISM: individual objects: HV Tau C - ISM: individual objects: RW Aur

\section{Introduction}

Herbig-Haro (HH) objects are the optically visible tracers of mass outflow from YSOs and are therefore ultimately powered by accretion (Cabrit et al. 1990; Hartigan et al. 1995). Over the years many bipolar $\mathrm{HH}$ outflows have been observed and most were found to be driven by embedded, low-mass sources of $\lesssim 1 M_{\odot}$. Initially it was assumed that their lengths were only a fraction of a parsec, however the discovery of a $\sim 1.4$ pc long outflow from RNO 43 (Ray 1987) hinted that this may not always be the case. In the mid 1990's it was realised that many of these outflows can have projected lengths much greater than 1 pc (Bally et al. 1996; Eislöffel \& Mundt 1997; Reipurth et al. 1997), reaching up to $\sim 11$ pc. Some well known examples are the HH 34 outflow consisting of $\mathrm{HH} 33$, $\mathrm{HH} 40$, HH 85, HH 126, HH 34N, HH 34, HH 34X, HH 173, HH 86, HH 87 and HH 88 which is 3 pc in projected length (Bally \& Devine 1994); the 5.9 pc long HH 401, HH 1, HH 2 and HH 402 outflow (Ogura 1995); and the HH 113/HH 111/HH311 outflow at $7.7 \mathrm{pc}$ in length (Reipurth et al. 1997).

It is not surprising that such outflows can attain these lengths when we consider that they have tangential velocities of between 50-200 $\mathrm{km} \mathrm{s}^{-1}$ (Devine et al. 1997; Reipurth et al. 1997) and the outflow phase, for even (low-mass) Class I sources, lasts at least $10^{5}$ years. In reality, it should be expected that most will attain parsec-scale lengths. The main

Send offprint requests to: F. McGroarty, e-mail: fmcg@cp.dias.ie observational hindrance in the past to observing them was the relatively small fields of view offered by most CCD cameras. With the advent of large CCD mosaics more and more parsecscale outflows have been discovered.

The morphology of parsec-scale outflows yields valuable information about their driving sources. They are, in effect, a fossil record of the mass-loss history of their source over their dynamical timescales $\left(\sim 10^{3}\right.$ to $\left.\sim 10^{5} \mathrm{yr}\right)$. They suggest, for example, quiescent phases between periods of violent mass ejection that give rise to the large $\mathrm{HH}$ complexes we see today. The morphology of an outflow can also indicate whether it is precessing and, if so, the rate of precession.

As mentioned earlier many of the parsec-scale outflows that have been observed to date are driven by young, Class I, low-mass YSOs. The classification scheme used here is based on the shape of the spectral energy distribution (SED) of the YSO from $10 \mu \mathrm{m}$ to $100 \mu \mathrm{m}$ (Lada \& Wilking 1984; Lada 1987). The SED of a YSO can be modelled as an approximate blackbody with an infrared excess longwards of $2 \mu \mathrm{m}$ due to circumstellar dust and gas. The infrared excess is very strong in the young, embedded Class I sources and is almost non-existent in the most evolved Class III sources. Here we observed a number of Class II low-mass sources - CTTSs. These CTTSs were not previously associated with parsec-scale outflows; in fact many were only known to drive "micro-jets" of the order of $\sim 5^{\prime \prime}$ to $40^{\prime \prime}(\$ 0.03 \mathrm{pc}$ at a distance of $140 \mathrm{pc}$ to the Taurus-Auriga Cloud). Although outflows from these more 
evolved sources are not nearly as spectacular as those from Class I YSOs, their sources are no longer surrounded by significant amounts of dust and so their outflows can be traced right back to the origin. These Class II sources are still actively accreting and ejecting matter, albeit at rates 10-100 times smaller than Class I sources (Hartigan et al. 1995). In this paper, we present a number of parsec-scale outflows from CTTSs and we investigate whether these CTTSs show evidence for having undergone FU Orionis-like outbursts, based on the fossil record of their outflows.

Details about the observations are given in Sect. 2. In Sect. 3 we report the discovery of parsec-scale outflows from five CTTSs in the Taurus-Auriga Cloud, at a distance of $140 \mathrm{pc}$ (Elias 1978; Wichmann et al. 1998). We also include serendipitous observations of a parsec-scale outflow from a less evolved Class I source. These results are discussed in Sect. 4 and our conclusions are presented in Sect. 5 .

\section{Observations}

Our data was acquired using the Wide Field Camera (WFC) on the $2.5 \mathrm{~m}$ Isaac Newton Telescope at El Observatorio del Roque de los Muchachos (La Palma, Canary Islands). The WFC consists of four thin-coated EEV CCDs each with $2048 \times 4100$, $15 \mu \mathrm{m} \times 15 \mu \mathrm{m}$ pixels. One pixel projects to 0 ' 33 on the sky. Three of the CCDs are positioned from north to south with their long axes adjoining. The fourth is attached to the west to form a square (34.2 wide) with its northwestern corner missing.

Our images were taken during two separate observing runs, the first between the 10th and 13th of February 2001, and the second between the 24th and 27th of November 2003. HH objects were identified using a number of narrowband emission line filters: $\mathrm{H} \alpha\left(\lambda_{c}=6568 \AA, \Delta \lambda(F W H M)=95 \AA\right)$ and $[\mathrm{SII}]\left(\lambda_{c}=6725 \AA, \Delta \lambda(F W H M)=80 \AA\right)$. To distinguish $\mathrm{HH}$ emission from reflection nebulosity, we also took broadband images in $I$ and $V$. Seeing in the images taken in February 2001 was moderate at $1^{\prime \prime}-2^{\prime \prime}$ as measured from the images. The seeing in the November 2003 images was better at $0{ }^{\prime} 9$ 1 '. 1 . Exposure times for the narrowband and broadband images were typically 30 and $10 \mathrm{~min}$ respectively. The data were reduced using standard IRAF reduction procedures. The sources observed here are CTTSs with previously known "micro-jets" or short outflows of $\lesssim 1^{\prime}$. All are in the Taurus-Auriga cloud, chosen for its abundance of CTTSs. Seven such sources were observed, with 5 of them - CW Tau, DG Tau, DO Tau, HV Tau C and RW Aur - revealing extended outflows of the order of $1 \mathrm{pc}$. No extended optical emission was found in the UY Aur and DP Tau outflows.

\section{Results}

\subsection{CW Tau}

CW Tau has a spectral type of K3 (Cohen \& Kuhi 1979), with $M_{\star}=1.40 M_{\odot}$ and $L_{\star}=2.6 L_{\odot}$ (Gomez de Castro 1993). A "micro-jet" (HH 220) propagating southeast from this source was discovered in optical images by Gomez de Castro (1993), with a gap of $\sim 1$ '. 3 between the source and the jet. A full
Table 1. Positions of the new HH objects found in this survey and their probable sources.

\begin{tabular}{llll}
\hline \hline Object & Source & $\alpha(\mathrm{J} 2000)$ & $\delta(\mathrm{J} 2000)$ \\
\hline HH 826 A & CW Tau & $04^{\mathrm{h}} 14^{\mathrm{m}} 17.8^{\mathrm{s}}$ & $+28^{\circ} 10^{\prime} 40^{\prime \prime}$ \\
HH 826 B & CW Tau & $04^{\mathrm{h}} 14^{\mathrm{m}} 19.8^{\mathrm{s}}$ & $+28^{\circ} 09^{\prime} 52^{\prime \prime}$ \\
HH 827 & CW Tau & $04^{\mathrm{h}} 14^{\mathrm{m}} 15.1^{\mathrm{s}}$ & $+28^{\circ} 03^{\prime} 55^{\prime \prime}$ \\
HH 826C & CW Tau & $04^{\mathrm{h}} 14^{\mathrm{m}} 15.3^{\mathrm{s}}$ & $+28^{\circ} 11^{\prime} 39^{\prime \prime}$ \\
HH 828 & CW Tau & $04^{\mathrm{h}} 14^{\mathrm{m}} 10.3^{\mathrm{s}}$ & $+28^{\circ} 14^{\prime} 54^{\prime \prime}$ \\
HH 829 A & CW Tau & $04^{\mathrm{h}} 14^{\mathrm{m}} 03.0^{\mathrm{s}}$ & $+28^{\circ} 25^{\prime} 36^{\prime \prime}$ \\
HH 829 B & CW Tau & $04^{\mathrm{h}} 14^{\mathrm{m}} 04.8^{\mathrm{s}}$ & $+28^{\circ} 26^{\prime} 53^{\prime \prime}$ \\
HH 829C & CW Tau & $04^{\mathrm{h}} 14^{\mathrm{m}} 07.3^{\mathrm{s}}$ & $+28^{\circ} 27^{\prime} 37^{\prime \prime}$ \\
HH 830 A & DG Tau & $04^{\mathrm{h}} 27^{\mathrm{m}} 37.3^{\mathrm{s}}$ & $+26^{\circ} 12^{\prime} 27^{\prime \prime}$ \\
HH 830 B & DG Tau & $04^{\mathrm{h}} 27^{\mathrm{m}} 44.8^{\mathrm{s}}$ & $+26^{\circ} 12^{\prime} 56^{\prime \prime}$ \\
HH 830C & DG Tau & $04^{\mathrm{h}} 27^{\mathrm{m}} 51.7^{\mathrm{s}}$ & $+26^{\circ} 15^{\prime} 33^{\prime \prime}$ \\
HH 831 A & DO Tau & $04^{\mathrm{h}} 39^{\mathrm{m}} 13.2^{\mathrm{s}}$ & $+26^{\circ} 13^{\prime} 48^{\prime \prime}$ \\
HH 831 B & DO Tau & $04^{\mathrm{h}} 39^{\mathrm{m}} 15.2^{\mathrm{s}}$ & $+26^{\circ} 13^{\prime} 55^{\prime \prime}$ \\
HH 832 & DO Tau & $04^{\mathrm{h}} 39^{\mathrm{m}} 02.0^{\mathrm{s}}$ & $+26^{\circ} 12^{\prime} 21^{\prime \prime}$ \\
HH 833 & HV Tau C & $04^{\mathrm{h}} 38^{\mathrm{m}} 44.0^{\mathrm{s}}$ & $+26^{\circ} 14^{\prime} 42^{\prime \prime}$ \\
HH 834 & $?$ & $04^{\mathrm{h}} 39^{\mathrm{m}} 05.9^{\mathrm{s}}$ & $+26^{\circ} 03^{\prime} 23^{\prime \prime}$ \\
HH 835 & RW Aur & $05^{\mathrm{h}} 07^{\mathrm{m}} 30.4^{\mathrm{s}}$ & $+30^{\circ} 27^{\prime} 11^{\prime \prime}$ \\
HH 836 A & DG Tau B & $04^{\mathrm{h}} 27^{\mathrm{m}} 13.5^{\mathrm{s}}$ & $+26^{\circ} 04^{\prime} 16^{\prime \prime}$ \\
HH 836 B & DG Tau B & $04^{\mathrm{h}} 27^{\mathrm{m}} 19.7^{\mathrm{s}}$ & $+26^{\circ} 03^{\prime} 07^{\prime \prime}$ \\
HH 837 & DG Tau B & $04^{\mathrm{h}} 27^{\mathrm{m}} 44.8^{\mathrm{s}}$ & $+26^{\circ} 00^{\prime} 49^{\prime \prime}$ \\
HH 838 & $?$ & $04^{\mathrm{h}} 26^{\mathrm{m}} 56.4^{\mathrm{s}}$ & $+26^{\circ} 05^{\prime} 58^{\prime \prime}$ \\
HH 839 & $?$ & $04^{\mathrm{h}} 27^{\mathrm{m}} 43.8^{\mathrm{s}}$ & $+26^{\circ} 04^{\prime} 35^{\prime \prime}$ \\
\hline
\end{tabular}

opening angle of $3.3^{\circ}$ is derived for this blueshifted jet at $3^{\prime \prime}$ from the source (Dougados et al. 2000). Long-slit spectroscopic observations by Hirth et al. (1994) showed the outflow to extend at least $4^{\prime \prime}-6$ " on either side of the source. The blueshifted HH 220 outflow is at a position angle (PA) of $144^{\circ} \pm 2^{\circ}$ with respect to CW Tau (Gomez de Castro 1993; Dougados et al. 2000) and we estimate the redshifted jet to be at $\sim 329^{\circ}$ with respect to $\mathrm{CW}$ Tau from the [SII] images of Dougados et al. (2000).

Our wide field images of the region around CW Tau (Fig. 1) reveal that this outflow is much more extended than just the HH 220 bipolar jet - see Table 2 for details of the newly discovered objects in the $\mathrm{CW}$ Tau outflow. Two knots are found to the south of CW Tau at 22" (HH 826 A) and 1'.27 (HH 826 B). $\mathrm{HH} 826 \mathrm{~A}$ is only seen in [SII] emission while HH $827 \mathrm{~B}$ is seen in both [SII] and $\mathrm{H} \alpha$. Both are at a PA of $\sim 153^{\circ}$ with respect to the source and are reasonably well aligned with the blueshifted HH 220 jet. Further out at 6.1 is HH 827 at a PA of $184^{\circ}$ with respect to CW Tau. Precession of the outflow may explain this directional change. HH 827 consists of a bright knot with a trail of emission stretching to the northeast and a fainter trail of emission to the southwest (see Fig. 3) and is much brighter and more extended in $\mathrm{H} \alpha$ than in [SII]. The total length of this object in $\mathrm{H} \alpha$ is $\sim 1$ '.8. It is possible that $\mathrm{HH} 827$ is not driven by CW Tau in which case IRAS $04113+2758$ and IRAS $04112+2803$ to the northeast and north of $\mathrm{HH} 827$ (marked in Fig. 1) are candidate sources, however the trail of emission from HH 827 doesn't point back to either of these. 
Table 2. Angular separations, PAs and spatial extent of newly discovered $\mathrm{HH}$ objects in the CW Tau region.

\begin{tabular}{llccl}
\hline \hline Object & Source & $\begin{array}{c}\text { Angular } \\
\text { separation }^{a}\end{array}$ & $\begin{array}{c}\text { PA }^{b} \\
{ }^{\circ}\end{array}$ & $\begin{array}{l}\text { Spatial } \\
\text { extent }^{c}\end{array}$ \\
\hline HH 826 A & CW Tau & 0.37 & 153 & $2^{\prime \prime} \times 2^{\prime \prime}$ \\
HH 826 B & CW Tau & $1 ! 27$ & 153 & $2^{\prime \prime} \times 5^{\prime \prime}$ \\
HH 827 & CW Tau & $6 ! 2$ & 184 & $1^{\prime} 8 \times 16^{\prime \prime}$ \\
HH 826C & CW Tau & 0.77 & 326 & $4^{\prime \prime} \times 3^{\prime \prime}$ \\
HH 828 & CW Tau & 4.52 & 342 & $3^{\prime \prime} \times 2^{\prime \prime}(\mathrm{e})^{d}$ \\
& CW Tau & 4.27 & 338 & $2^{\prime \prime} \times 3^{\prime \prime}(\mathrm{m})^{d}$ \\
& CW Tau & 4.33 & 334 & $5^{\prime \prime} \times 3^{\prime \prime}(\mathrm{w})^{d}$ \\
HH 829 A & CW Tau & 14.9 & 348 & $29^{\prime \prime} \times 5^{\prime \prime}$ \\
HH 829 B & CW Tau & 16.12 & 351 & $3^{\prime \prime} \times 4^{\prime \prime}$ \\
HH 829C & CW Tau & $16 ! 8$ & 353 & $3^{\prime \prime} \times 6^{\prime \prime}$ \\
\hline
\end{tabular}

${ }^{a}$ Angular distance from the presumed source.

${ }^{b}$ PA with respect to the presumed source.

${ }^{c}$ Width and length of the object respectively.

${ }^{d}$ The three knots in HH 828 (see Fig. 2) are labelled here as the eastern-most knot (e), the middle knot (m) and the western-most knot $(\mathrm{w})$.

If $\mathrm{HH} 827$ is driven by $\mathrm{CW}$ Tau then the projected length of the blueshifted outflow is 7'.08 $(0.29 \mathrm{pc})$.

Our [SII] images show that the redshifted HH 220 jet extends to $\sim 46^{\prime \prime}$ from CW Tau at a PA of $326^{\circ}$ (Fig. 1). The jet first extends to $\sim 9^{\prime \prime}$ from the source, then there is a gap of almost $20^{\prime \prime}$ where the jet is too faint to be seen. It becomes visible again for a distance of $\sim 17^{\prime \prime}$ before terminating in the bright knot HH 826 C. Further out a trio of knots (HH 828) are found at $\sim 4$. 3 from CW Tau and are only seen in [SII] (Figs. 1 and 2). The most western knot is at a PA of $334^{\circ}$ from CW Tau and the most eastern is at $342^{\circ}$. There are three known IRAS sources in the vicinity of $\mathrm{HH} 828$ (marked in Fig. 1) and it is possible that the source of the HH 828 knots is one of these or CW Tau itself. Proper motion studies would help distinguish between these possibilities.

There is a distance of $\sim 10$ '. (0.44 pc) between HH 828 and the next $\mathrm{HH}$ object along this direction, $\mathrm{HH} 829 \mathrm{~A}$ (Figs. 1 and 4). HH $829 \mathrm{~A}, \mathrm{~B}$ and $\mathrm{C}$ are $14 \mathbf{1}^{\prime} .9,16^{\prime} .12$ and $16^{\prime} .8$ at $348^{\circ}$, $351^{\circ}$ and $353^{\circ}$ from CW Tau respectively. The edge of this complex is approximately $37^{\prime \prime}$ from the northern edge of our field of view so it is possible that further emission is present beyond this. While $\mathrm{HH} 829 \mathrm{~A}$ is comparable in brightness in both $\mathrm{H} \alpha$ and [SII] emission, knots B and $\mathrm{C}$ are brighter in [SII] and $\mathrm{H} \alpha$ respectively (Fig. 4). IRAS $04111+2820$ (slightly outside the field of view of Fig. 1) is $\sim 1.5$ at a PA of $78^{\circ}$ from $\mathrm{HH} 829 \mathrm{C}$ but is highly unlikely to be driving this set of colinear knots given their position with respect to this source. The projected length of this assumed CW Tau redshifted outflow is $0.69 \mathrm{pc}(16 ! 8)$. This gives a total projected length of the CW Tau blue - and redshifted outflows of $\sim 0.98 \mathrm{pc}$.

The variation in PA in the blueshifted outflow is $40^{\circ}$ and is $24^{\circ}$ in the redshifted outflow. This change in direction gives the extended outflow an inverted "S" shape. Similar morphologies are seen in many large-scale outflows from less evolved
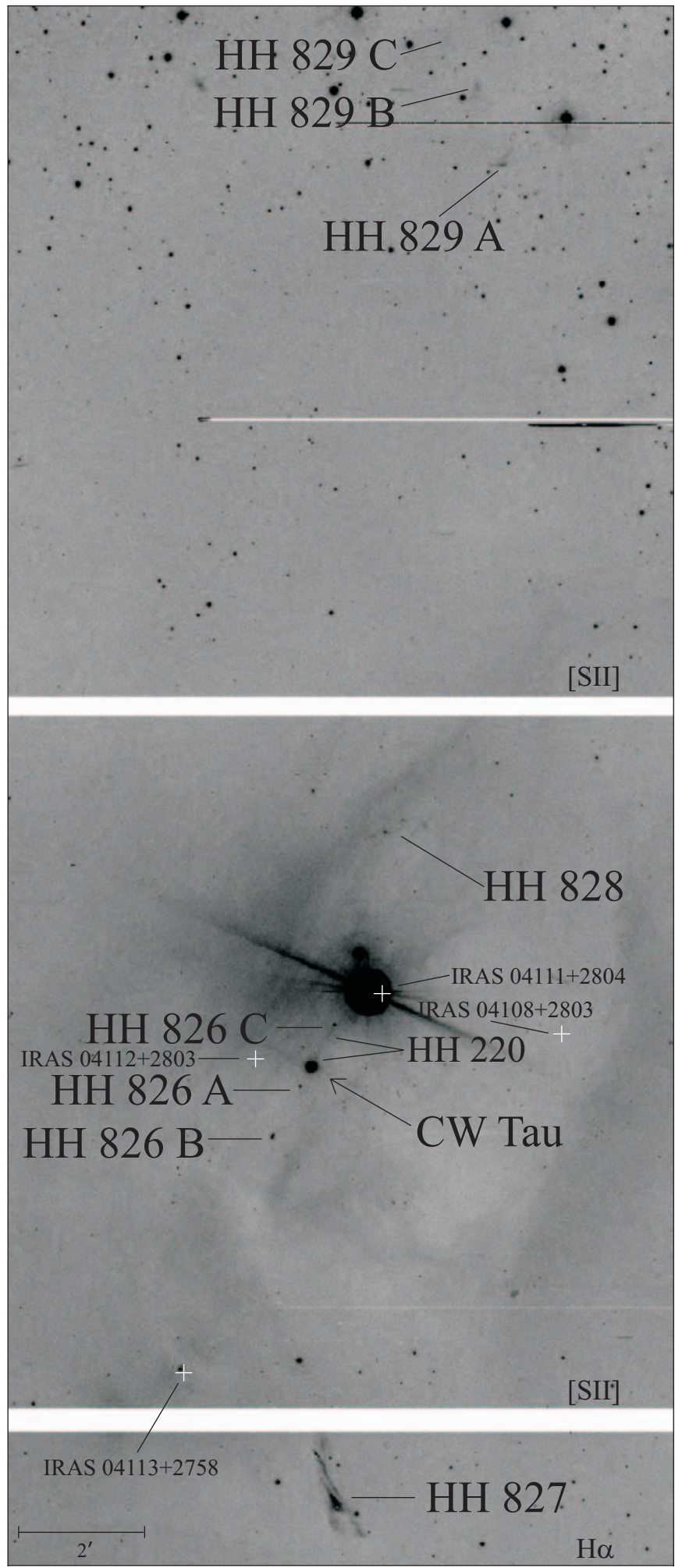

Fig. 1. CW Tau: mosaic of the entire CW Tau outflow. The top and middle frames are in [SII] and the bottom frame is in $\mathrm{H} \alpha$ as $\mathrm{HH} 827$ is much stronger in $\mathrm{H} \alpha$. All known IRAS sources in the region are marked with white crosses. The individual knots can be seen more clearly in Figs. 2 to 4. Any information in the gaps between the CCDs (white strips) is lost. The northern edge of this image is the northern edge of the field of view of the CCD Mosaic. Note that the linear object to the east of HH $829 \mathrm{~B}$ is an asteroid trail. For all images in this paper, North is up and West is to the right. 

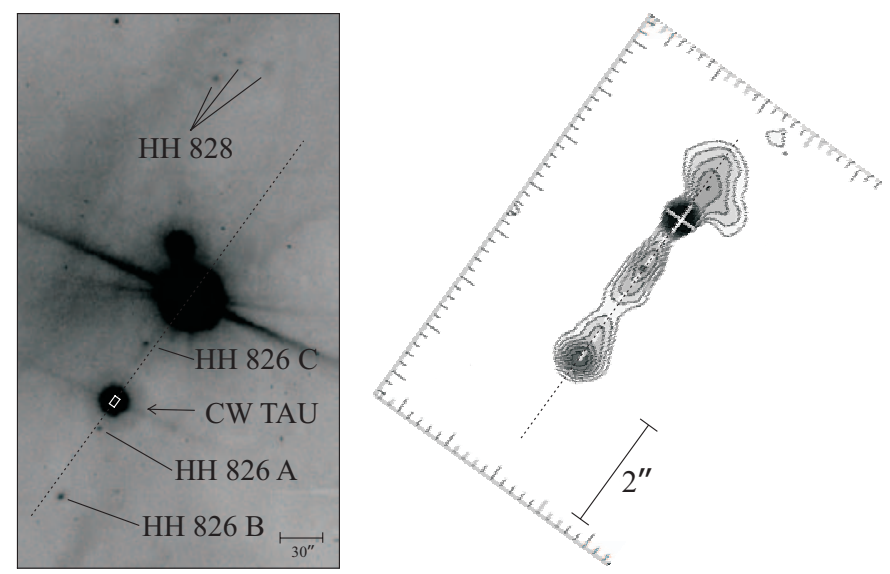

Fig. 2. CW Tau: left: our [SII] image showing CW Tau and the newly discovered HH 826 and HH 828 knots. The small white box superimposed on CW Tau marks the region shown on the right. Right: CW Tau and the HH220 jet taken from Dougados et al. (2000). A dotted line shows the PA of $144^{\circ}$ determined by Dougados et al. (2000) and a similar line at $144^{\circ}$ is superimposed on our [SII] image to show the slight change in direction of the outflow.

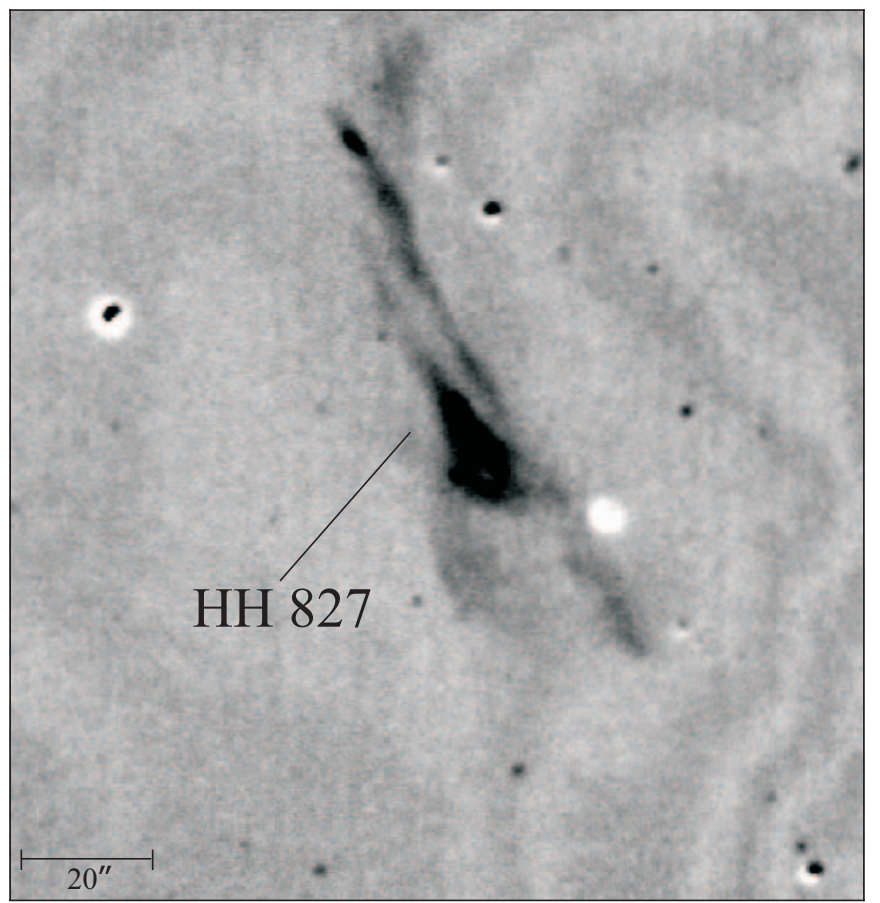

Fig. 3. $\mathrm{HH} 827 \mathrm{H} \alpha$ : continuum subtracted $\mathrm{H} \alpha$ image of $\mathrm{HH} 827$ to the south of CW Tau. The trail of emission to the northeast is clearly seen and the fainter trail to the southwest extends for $\sim 31^{\prime \prime}$ from the southern end of the bright knot.

low-mass sources (see Sect. 4.1) and suggests outflow precession. The change in direction is approximately symmetrical about CW Tau which substantiates our suggestion that both HH 827 and HH 829 may be part of this outflow. CW Tau is surrounded by a dark cloud (Fig. 1) so the majority of the outflow is presumably obscured by this cloud. It is interesting to note however that the more distant objects in this outflow, HH 827 and $\mathrm{HH} \mathrm{829,} \mathrm{are} \mathrm{found} \mathrm{at} \mathrm{the} \mathrm{cloud} \mathrm{edges.}$
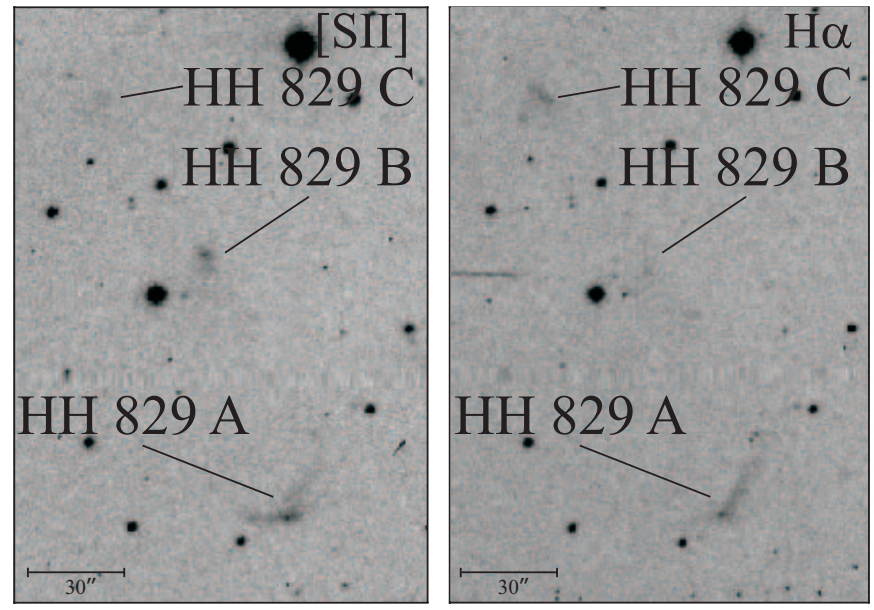

Fig. 4. HH 829: image of HH 829 A, B and C to the north of CW Tau in [SII] (left) and $\mathrm{H} \alpha(r i g h t)$. $\mathrm{HH} 829 \mathrm{C}$ is stronger in $\mathrm{H} \alpha$ emission and $\mathrm{HH} 829 \mathrm{~B}$ is stronger in $[\mathrm{SII}]$ emission, while $\mathrm{HH} 829 \mathrm{~A}$ is comparable in both.

\subsection{The DG Tau region}

DG Tau is a low luminosity star with $L_{\star} \simeq 8 L_{\odot}$ (Cohen \& Kuhi 1979) and was one of the first TTSs to be associated with an optical jet (HH 158), noted by Mundt \& Fried (1983). HST/STIS observations show HH 158 to be at a PA of $223^{\circ}$ with respect to DG Tau (Bacciotti et al. 2002) and that the blueshifted HH 158 "micro-jet" can be traced to within $\sim 0$ ' 1 from the star (Bacciotti et al. 2000). Larger scale studies show the HH 158 outflow to extend to $\sim 11^{\prime \prime}$, with a number of resolved knots (Eislöffel \& Mundt 1998). A redshifted jet $\sim 1^{\prime \prime}$ in length is detected $\sim 0$ ' 45 from the source (Lavalley et al. 1997). There is a semi-circular nebula associated with DG Tau (Mundt et al. 1987) which HST data resolves as three nested arcs of reflection at $2^{\prime \prime}, 5^{\prime \prime}$ and 10" from DG Tau (Stapelfeldt et al. 1997). The axis of symmetry of all three arcs is $\sim 225^{\circ}$ with respect to DG Tau which is well aligned with the PA of $\mathrm{HH} 158$, suggesting that these arcs are the illuminated edges of a cavity carved by the blueshifted jet.

DG Tau B is located $47^{\prime \prime}$ south and $28^{\prime \prime}$ west of DG Tau and is a low luminosity Class $I$ source with $L_{\star}=0.88 L_{\odot}$ (Jones \& Cohen 1986). It is the driving source of an optical jet (HH 159) also found by Mundt \& Fried (1983) and seen to be bipolar by Jones \& Cohen (1986). HST images have resolved DG Tau B as a compact bipolar reflection nebula with no optically visible star (Stapelfeldt et al. 1997). The eastern lobe of the reflection nebula is "V" shaped and the PA of its axis of symmetry is $122^{\circ}$ (Padgett et al. 1999). The 56" redshifted jet and 15" blueshifted jet have been observed both in imaging mode (Mundt \& Fried 1983; Mundt et al. 1991; Eislöffel \& Mundt 1998) and spectroscopically (Jones \& Cohen 1986; Mundt et al. 1987; Eislöffel $\&$ Mundt 1998). Position angles of $122^{\circ}$ for the blueshifted jet and $296^{\circ}$ for the redshifted jet were determined by Mundt et al. (1991). However examination of our images would suggest that the PA of the blueshifted jet is closer to $116^{\circ}$ i.e. the two jets are diametrically opposed as one might expect. In either case, the blueshifted jet is approximately coincident with the axis of symmetry of the reflection nebula, suggesting that the " $\mathrm{V}$ " 

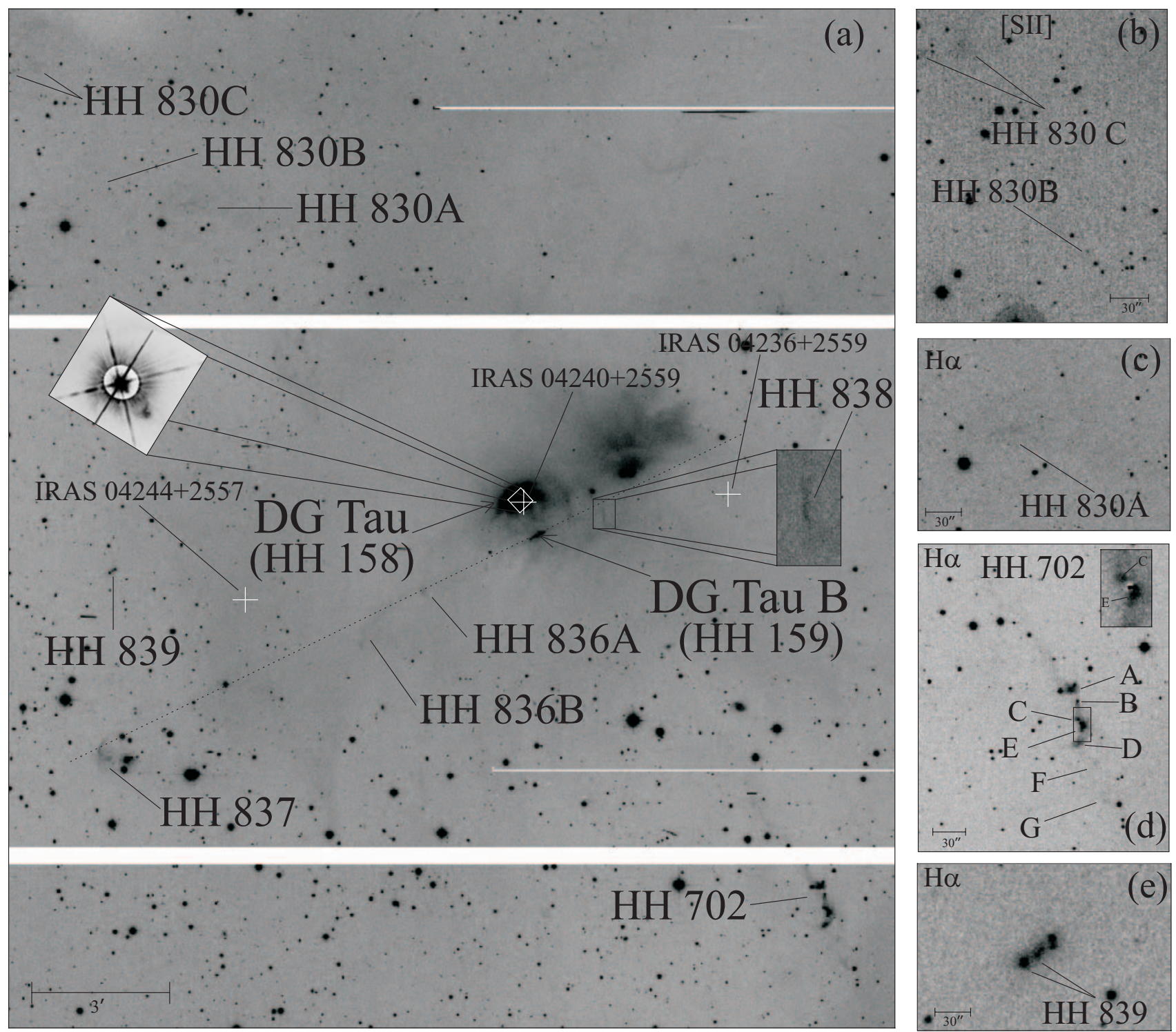

Fig. 5. DG Tau: a) mosaic image of the region surrounding DG Tau and DG Tau B in H $\alpha$ : the new objects discovered in both the DG Tau outflow (HH 830) and the DG Tau B outflow (HH 836 and HH 837) are indicated here. HH 838 and HH 839 appear to be separate outflows in this region. A HST image of HH 158 is included here as an inset and has been rotated such that north is up. This inset is $21^{\prime \prime} 7$ by 22 '. 05 . The dotted line through the centre of the image is at $116^{\circ}$ and marks the blueshifted DG Tau B outflow. The three known IRAS sources in the region are marked with white crosses. b) HH $830 \mathrm{~B}$ and C in [SII]: HH $830 \mathrm{C}$ can be seen here to be two distinct regions of emission, and HH $830 \mathrm{~B}$ is a small knot. Note that the left hand edge of the image is actually the eastern edge of our field of view of the CCD mosaic so it is quite possible that $\mathrm{HH} 830 \mathrm{C}$ is more extended. c) $\mathrm{HH} 830 \mathrm{~A}$ in $\mathrm{H} \alpha$ : HH $830 \mathrm{~A}$ is a faint, linear emission object extending $28^{\prime \prime}$ and dips southwards towards the western edge for another 16". d) HH 702 in H $\alpha$ : an image taken in February 2001 is used here as it shows the whole of HH 702 in one CCD field. Knots A, B, C and D (discovered by Sun et al. 2003) can clearly be seen. The newly discovered Knot E and fainter Knots F and G are also marked here. There is a star to the north of Knot E but this knot can be seen clearly in the H $\alpha$ continuum subtracted image (inset). e) HH 839 in $\mathrm{H} \alpha$.

delineates the walls of a cavity cleared by it. The redshifted jet passes through the centre of a well collimated redshifted CO emission lobe (Mitchell et al. 1994, 1997).

The most spectacular object in this outflow is HH702 an $\sim 4^{\prime}$ long shock system to the southwest of DG Tau. This object has already been noted by Sun et al. (2003), however our $\mathrm{H} \alpha$ images show more detail than the [SII] image of Sun et al. (2003). HH 702 consists of five bright knots (Fig. 5d) (one of which (E) was not noted by Sun et al. 2003), two fainter knots ( $F$ and $G$ ) and a trail of emission to the northeast. From our images, the trail of emission is first seen 7.9 from DG Tau at a PA of $218^{\circ}$, pointing back towards DG Tau, and stretches a distance of 2.75 to Knot A. Knot A is an intense emission knot at 10'6 from DG Tau, with a star directly to the east. Knots C and $\mathrm{D}$ are on either side of a star and can be seen more clearly in the continuum subtracted image inset in Fig. 5d. While a number of IRAS sources in the vicinity have been previously suggested as possible sources for HH 702 (Sun et al. 2003), we think it is more likely that this object is driven by DG Tau given the alignment between HH 702 and HH 158. The most distant 
Table 3. Angular separations, PAs and spatial extent of newly discovered $\mathrm{HH}$ objects in the DG Tau and DG Tau B region.

\begin{tabular}{|c|c|c|c|c|}
\hline Object & Source & $\begin{array}{c}\text { Angular } \\
\text { separation }^{a}\end{array}$ & $\begin{array}{c}\mathrm{PA}^{b} \\
{ }^{\circ}\end{array}$ & $\begin{array}{l}\text { Spatial } \\
\text { extent }^{c}\end{array}$ \\
\hline $\mathrm{HH} 830 \mathrm{~A}$ & DG Tau & 9.6 & 48 & $5^{\prime \prime} \times 44^{\prime \prime}$ \\
\hline НH 830 B & DG Tau & $11 ! 3$ & 48 & $2^{\prime \prime} \times 2^{\prime \prime}$ \\
\hline \multirow[t]{2}{*}{ HH $830 \mathrm{C}$} & DG Tau & $14 ! 1$ & 47 & $21^{\prime \prime} \times$ \\
\hline & & $14 ! 4$ & 50 & $6^{\prime \prime} \times$ \\
\hline HH 836 A & DG Tau B & $2 ! 6$ & 116 & $14^{\prime \prime} \times$ \\
\hline НH 836 B & DG Tau B & 4.4 & 122 & $12^{\prime \prime} \times 3^{\prime \prime}$ \\
\hline \multirow[t]{2}{*}{ HH 837} & DG Tau B & $10 ! 4$ & 117 & $21^{\prime \prime} \times 32^{\prime \prime d}$ \\
\hline & & $10^{\prime} 4$ & 117 & $19^{\prime \prime} \times 18^{\prime \prime e}$ \\
\hline HН 838 & $?$ & & & $43^{\prime \prime} \times \quad 4^{\prime \prime}$ \\
\hline HН 839 & $?^{f}$ & & 126 & $3^{\prime \prime} \times 10^{\prime \prime}$ \\
\hline
\end{tabular}

${ }^{a}$ Angular distance from the presumed source.

${ }^{b}$ PA with respect to the presumed source.

${ }^{c}$ Width and length of the object respectively.

${ }^{d}$ The morphology of $\mathrm{HH} 837$ is different in $\mathrm{H} \alpha$ and [SII] emission; this is the width and length of this object in $\mathrm{H} \alpha$.

${ }^{e}$ The morphology of HH 837 is different in $\mathrm{H} \alpha$ and [SII] emission; this is the width and length of this object in [SII].

${ }^{f}$ The source of this outflow appears to be a stellar object at its base (see Sect. 3.2).

optically visible object in the blueshifted outflow, Knot $\mathrm{G}$ of HH 702 is 12 .32 from DG Tau, which is 0.5 pc in projected length at a distance of $140 \mathrm{pc}$ to the Taurus-Auriga cloud.

Our observations reveal a new HH complex (HH 830) in the DG Tau outflow, details of which are given in Table 3. HH 830 is a faint complex 9.6 to the northeast of DG Tau and consists of three separate emission objects A-C (Fig. 5c). HH $830 \mathrm{~A}$ is a 44" long linear object orientated east/west and dips southwards towards its western edge. HH $830 \mathrm{~B}$ is $\sim 11$ ! 3 from DG Tau and is brighter than HH $830 \mathrm{~A}$ but is much smaller in size (Fig. 5b). HH $830 \mathrm{C}$ is the most distant optically visible object in this outflow and appears to be two separate emission regions at 14.1 (at PA of $47^{\circ}$ ) and 14.4 (at PA of $50^{\circ}$ ) from the source (Fig. 5b). This object is at one edge of our image and there there might be more emission further east. The HH 830 knots are comparable in $\mathrm{H} \alpha$ and [SII] emission. Both $\mathrm{HH} 830 \mathrm{~A}$ and the centre of $\mathrm{HH} 830 \mathrm{C}$ are along a PA of $\sim 48^{\circ}$ with respect to DG Tau. Thus they are reasonably well aligned with the redshifted jet which we estimate to be at $44^{\circ}$ from the images of Lavalley et al. (1997). HH $830 \mathrm{~B}$ is slightly off this axis, at a PA of $52^{\circ}$. The total length of the redshifted outflow is 14.4 i.e. $0.59 \mathrm{pc}$, giving the total projected length of the DG Tau bipolar outflow to be $1.09 \mathrm{pc}$.

The outflow direction changes by $5^{\circ}$ in the blueshifted outflow and $8^{\circ}$ in the redshifted outflow giving the extended outflow a "C" shaped morphology, with DG Tau at the apex of the "C". Similar morphologies have been noted in a small number of large-scale outflows from less evolved sources, for example the HH 366 outflow in Barnard 5 (Bally et al. 1996). This morphology may be analogous to the head-tail extragalactic radio sources where a curved morphology is created between the radio source (head) and the bipolar jet (tails) due to the
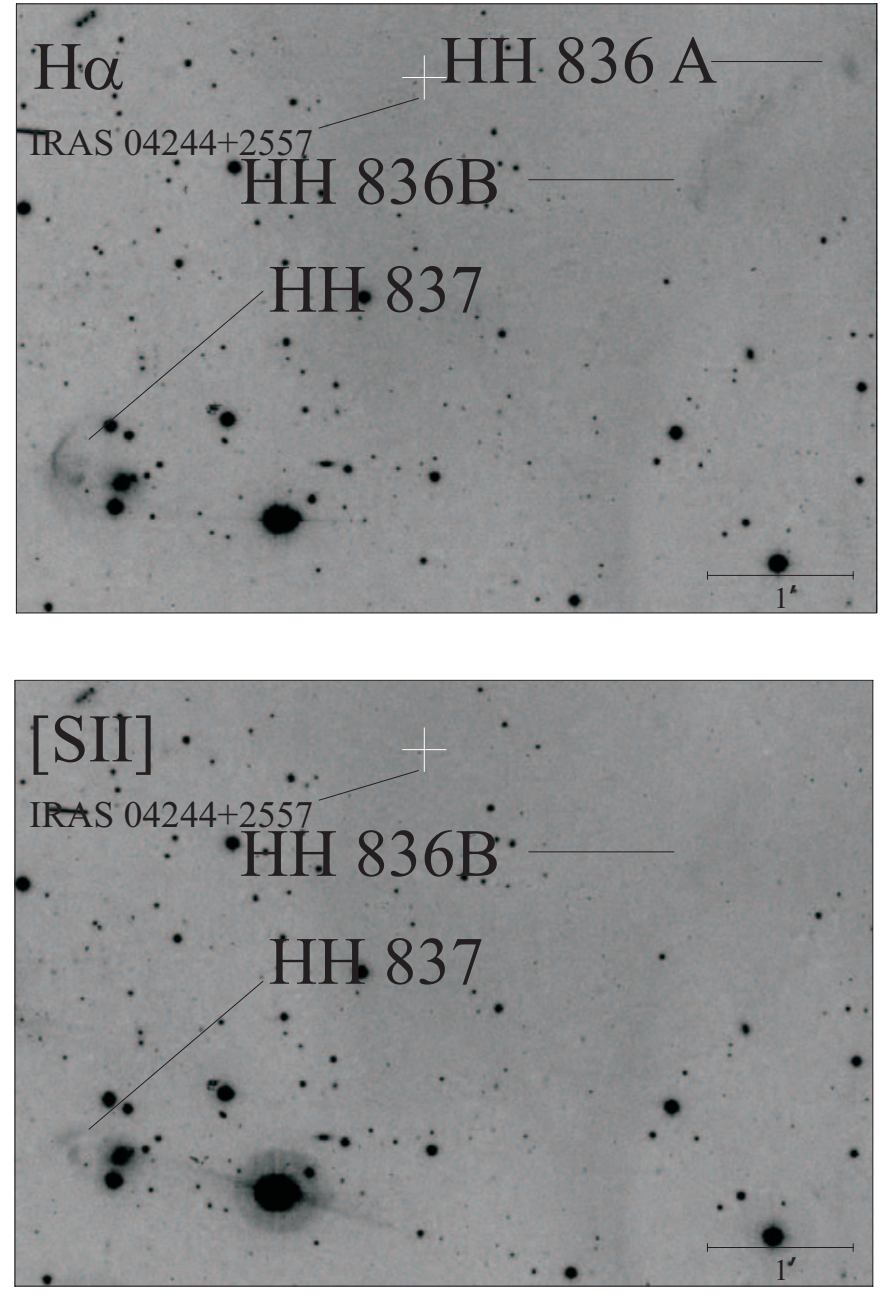

Fig. 6. HH 836 and HH 837: the objects to the southeast of DG Tau B are shown here in both $\mathrm{H} \alpha$ and [SII]. See Fig. 5a for the extended DG Tau B outflow. HH 836 A is only seen in $\mathrm{H} \alpha$, and $\mathrm{HH} 836 \mathrm{~B}$ is stronger in $\mathrm{H} \alpha$ than in [SII]. There is a distinct difference between $\mathrm{HH} 837$ in $\mathrm{H} \alpha$ and [SII]. The nearby IRAS source, which is also marked in Fig. 5a, is marked here.

motion of the source through the ISM (Edge \& Rottgering 1995; Valentijn 1981). In the YSO case, typical stellar velocities of around $5-10 \mathrm{~km} \mathrm{~s}^{-1}$ with respect to the parent cloud, combined with jet velocities of approximately $150 \mathrm{~km} \mathrm{~s}^{-1}$, might produce a similar effect.

Our observations also reveal two faint new objects to the southeast of DG Tau B, with a third more evident one further out in the same direction (Figs. 5 and 6), details of which are given in Table 3. HH $836 \mathrm{~A}$ is at $\sim 2$ ' 6 from the source and is seen only in $\mathrm{H} \alpha$. HH 836 B is at 4.4 from DG Tau B and is seen in both $\mathrm{H} \alpha$ and [SII]. A trail of emission appears to curve back towards $\mathrm{HH} 836 \mathrm{~A}$ from $\mathrm{HH} 836 \mathrm{~B}$ in the $\mathrm{H} \alpha$ image. These $\mathrm{HH}$ objects are quite faint and diffuse. The most distant optically visible object in the blueshifted outflow is $\mathrm{HH} 837$ at 10.'4 from DG Tau B. In [SII] this object appears to have a "V" shaped morphology pointing eastwards. In $\mathrm{H} \alpha$ it is much stronger and is clearly bow shaped with some emission over-lapping the [SII] emission to the west of the bow (Fig. 6). There is no indication of this bow shock in the [SII] emission. Both HH $836 \mathrm{~A}$ 
and $\mathrm{HH} 837$ are at a PA of $116^{\circ}$ and $117^{\circ}$ respectively with respect to DG Tau B and are well aligned with the blueshifted $\mathrm{HH} 159$ jet. HH $836 \mathrm{~B}$ is slightly off this axis at $122^{\circ}$ suggesting that the direction of the blueshifted outflow has only varied slightly over the 10'. 4 distance from the source. Although IRAS $04244+2557$ is a possible driving source for some of these HH objects (see Fig. 5) it seems more than coincidental that all three are aligned with the DG Tau B jet. The length of this outflow from the redshifted $\mathrm{HH} 159$ jet to $\mathrm{HH} 837$ is 11 '.3 which is a projected length of $0.46 \mathrm{pc}$. It is interesting to note that the opening angle for the blueshifted jet was initially estimated to be $\sim 17^{\circ}$ i.e. almost four times that of the redshifted jet at $\sim 4.5^{\circ}$ (Mundt et al. 1991). Our images of the extended blueshifted outflow show that it has clearly recollimated; the extended opening angle (to $\mathrm{HH} 837$ ) is now $3^{\circ}$.

Two other $\mathrm{HH}$ objects are found in this region which do not appear to be related to either the DG Tau or the DG Tau B outflows. HH 838 is 1.6 at a PA of $286^{\circ}$ from DG Tau B and is a faint, $43^{\prime \prime}$ by $\sim 4^{\prime \prime}$ linear HH object running north/south (Fig. 5) seen only in $\mathrm{H} \alpha$ emission. All known IRAS sources in the regions are marked in Fig. 5 but none of these appear to be a likely driving source for $\mathrm{HH} 838$. Our images do not show any other $\mathrm{HH}$ emission aligned with $\mathrm{HH} 838$. The second $\mathrm{HH}$ object, HH 839 to the east of DG Tau B, consists of three "knots" (seen clearly in Fig. 5e), of which the most northwestern one appears to be a mixture of continuum and $\mathrm{HH}$ emission and is probably the location of the source driving the outflow. The other two knots which are at a PA of $126^{\circ}$ with respect to this assumed source appear to be an $\sim 10^{\prime \prime}$ long outflow and are equally strong in $\mathrm{H} \alpha$ and [SII]. This outflow is approximately parallel to the DG Tau B outflow. HH 839 is quite bright in our $I$ band images so it possibly contains some reflection nebulosity as well. We can find no other obvious driving source for this outflow.

\subsection{The DO Tau and HV Tau region}

DO Tau is a CTTS associated with an arc-like nebula. A bipolar jet (HH 230) extending for approximately $2^{\prime \prime}$ to $4^{\prime \prime}$ was observed spectroscopically from DO Tau by Hirth et al. (1994) with the redshifted jet at a PA of $\sim 70^{\circ}$ (Hirth et al. 1994). DO Tau is an M0 star (Herbig \& Bell 1988) with a mass in the range $0.3-0.7 M_{\odot}$ and an age of about $1.6 \times 10^{5}$ years (Beckwith et al. 1990; Hartigan et al. 1995). To the east of DO Tau is the triple system HV Tau consisting of a close binary, A and B (Simon et al. 1996), and a third component $C \sim 4$ " northeast of the close binary. HV Tau $\mathrm{C}$ is an actively accreting CTTS (Woitas \& Leinert 1998) and a bipolar "micro-jet" $\sim 1$ ".5 in length has recently been observed emerging from it (Stapelfeldt et al. 2003). We have estimated the PA of its blueshifted jet to be at $25^{\circ}$ with respect to the source from the images of Stapelfeldt et al. (2003).

Five $\mathrm{HH}$ objects were found in this region and are marked in Fig. 7a with details given in Table 4. Neither the DO Tau nor the HV Tau $\mathrm{C}$ jets are seen in our optical images because of the bright nebulosity around both stars. HH 831 is found roughly northeast of DO Tau and is seen in both [SII] and $\mathrm{H} \alpha$ although
Table 4. Angular separations, PAs and spatial extent of newly discovered $\mathrm{HH}$ objects in the DO Tau and HV Tau C region.

\begin{tabular}{|c|c|c|c|c|}
\hline Object & Source & $\begin{array}{c}\text { Angular } \\
\text { separation }^{a}\end{array}$ & $\begin{array}{c}\mathrm{PA}^{b} \\
1^{\circ}\end{array}$ & $\begin{array}{l}\text { Spatial } \\
\text { extent }^{c}\end{array}$ \\
\hline HH $831 \mathrm{~A}$ & DO Tau & $10^{\prime} .8$ & 74 & $4^{\prime \prime} \times 52^{\prime \prime}$ \\
\hline HH 831 B & DO Tau & $11^{\prime}$ & 74 & $14^{\prime \prime} \times 8^{\prime \prime}$ \\
\hline НН 832 & DO Tau & $7: 7$ & 78 & $4^{\prime \prime} \times 29^{\prime \prime}$ \\
\hline HH 833 & HV Tau C & 4.6 & 25 & $10^{\prime \prime} \times 8^{\prime \prime}$ \\
\hline HH 834 & $?$ & & & $42^{\prime \prime} \times 6^{\prime \prime}$ \\
\hline
\end{tabular}

${ }^{a}$ Angular distance from the presumed source.

${ }^{b} \mathrm{PA}$ with respect to the presumed source.

${ }^{c}$ Width and length of the object respectively.

there are morphological differences between these two images. HH 832 and HH 833 are also seen to the northeast of DO Tau however they are only visible in [SII]. HH 834 is the only object to the southeast and is seen only in $\mathrm{H} \alpha$.

In $\mathrm{H} \alpha$ images (Fig. 9) $\mathrm{HH} 831 \mathrm{~A}$ is a 52" long structure consisting of three small joined arcs and is fainter towards the northwest and HH $831 \mathrm{~B}$ is a diffuse knot. In [SII] images (Fig. 8) HH831 A is seen as two separate emission regions, the most distant of which is at 10'8 from DO Tau. HH $831 \mathrm{~B}$ contains a number of bright emission regions in [SII], more than are seen in $\mathrm{H} \alpha$ and is at a distance of $11^{\prime}$ from DO Tau. HH $831 \mathrm{~B}$ is only $\sim 36^{\prime \prime}$ from the eastern edge of our field of view so it is very possible that there is more emission in this direction. HH 831 is at a PA of $\sim 74^{\circ}$ with respect to DO Tau and is well aligned with the redshifted jet.

HH 832 (Fig. 8) is seen only in [SII] and is at $78^{\circ}$ and $7 ! 7$ from DO Tau. It is an $\sim 6^{\prime \prime}$ long linear object with faint emission to the northeast. There is a nearby trail from a passing satellite.

$\mathrm{HH} 833$ (Fig. 7a) is a diffuse region 4.'6 from HV Tau C at a PA of $25^{\circ}$ and is seen only in [SII]. HH 705, to the north of HH 831, is a complex object containing a number of bright emission regions (Figs. $7 \mathrm{~b}$ and $7 \mathrm{c}$ ). This object was discovered by Sun et al. (2003) however our images show it to be more intense and complex than their [SII] images. This object may be a bow shock, with faint emission trailing to the southeast and southwest forming the wings and there is a 1.3 long trail to the south. Its total width, as measured between the edges of the wings, is $\sim 1^{\prime}$ and its total length is 1 '.9. $^{\prime}$ HH 834 is very faint and seen only in $\mathrm{H} \alpha$ (Fig. 7d). It is linear and has a total length of $42^{\prime \prime}$.

The locations of these new objects in relation to previously known outflows suggests possible links. $\mathrm{HH} 230$ is at $\sim 70^{\circ}$ with respect to DO Tau so it is likely that $\mathrm{HH} 831$ at $74^{\circ}$, and possibly $\mathrm{HH} 832$ at a PA of $78^{\circ}$, are part of the same outflow. There is a gap of $\sim$ !.7 (0.43 pc) between the $4^{\prime \prime}$ redshifted $\mathrm{HH} 230$ jet and HH 832 where no $\mathrm{HH}$ emission is seen. If $\mathrm{HH} 831$ and $\mathrm{HH} 832$ are driven by DO Tau then the length of the redshifted outflow is $\sim 11$ '.07 $(0.45 \mathrm{pc})$.

Considering its alignment with the previously known "micro-jet" from HV Tau C, it is most likely that HH 833 is driven by this source. HH 705 could also be part of this outflow. Both the blueshifted jet from HV Tau $\mathrm{C}$ and $\mathrm{HH} 833$ are at $25^{\circ}$ 

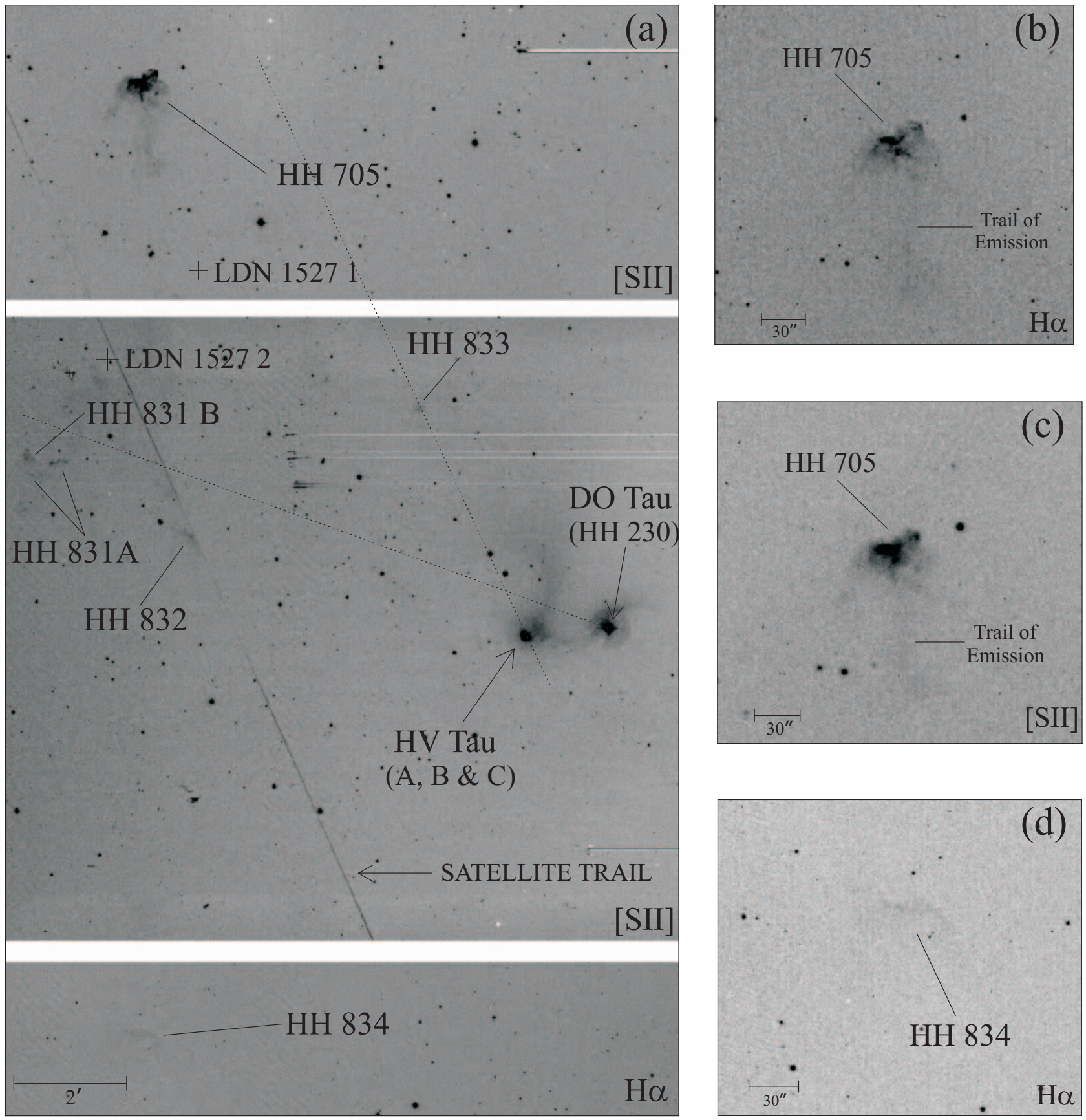

Fig. 7. DO Tau/HV Tau: a) mosaic of the DO Tau and HV Tau C regions showing the newly discovered HH objects HH 831-HH 834. The positions of candidate sources for these HH objects are marked. HH 230 is not seen in our optical images because of the DO Tau nebulosity, however the dashed line from DO Tau is at $70^{\circ}$ marking the direction of the redshifted jet. There is also a dashed line at $25^{\circ}$ from $\mathrm{HV}$ Tau $\mathrm{C}$ marking the PA of its blueshifted "micro-jet". HH 831 and HH 832 can be seen more clearly in Figs. 8 and 9. b) HH 705 in H $\alpha$ emission. c) HH 705 in [SII] emission. d) HH 834 in $\mathrm{H} \alpha$. Note that HH 834 is not seen in [SII] emission.

with respect to $\mathrm{HV}$ Tau $\mathrm{C}$ whilst $\mathrm{HH} 705$ is at $\sim 36^{\circ}$ with respect to this source at a distance of $12^{\prime}$ from it. HH 705 is $\sim 7$ '.7 from $\mathrm{HH} 833$ and it is possible that the outflow direction could have changed by $11^{\circ}$ over this distance. The southern trail of emission from $\mathrm{HH} 705$ might suggest that its driving source is situated to its south rather than southwest. In this case the radio sources LDN 15271 or LDN 15272 in LDN 1527 (Anglada et al. 1992) are possible driving sources (as suggested by Sun et al. (2003), however it is unlikely that LDN 15271 could generate such a large object so nearby. We think HV Tau C is the most likely source of this object. Assuming $\mathrm{HH} 833$ and HH 705 are driven by HV Tau C, the projected length of this outflow is $0.49 \mathrm{pc}\left(12^{\prime}\right)$. At present there is no obvious driving source for $\mathrm{HH} 834$.

\subsection{RW Aur}

RW Aur is a hierarchical triple system, with component A about 1." 4 at a PA of $76^{\circ}$ from the close binary B and C (Ghez et al. 1993). Both RW Aur A and B are actively accreting 


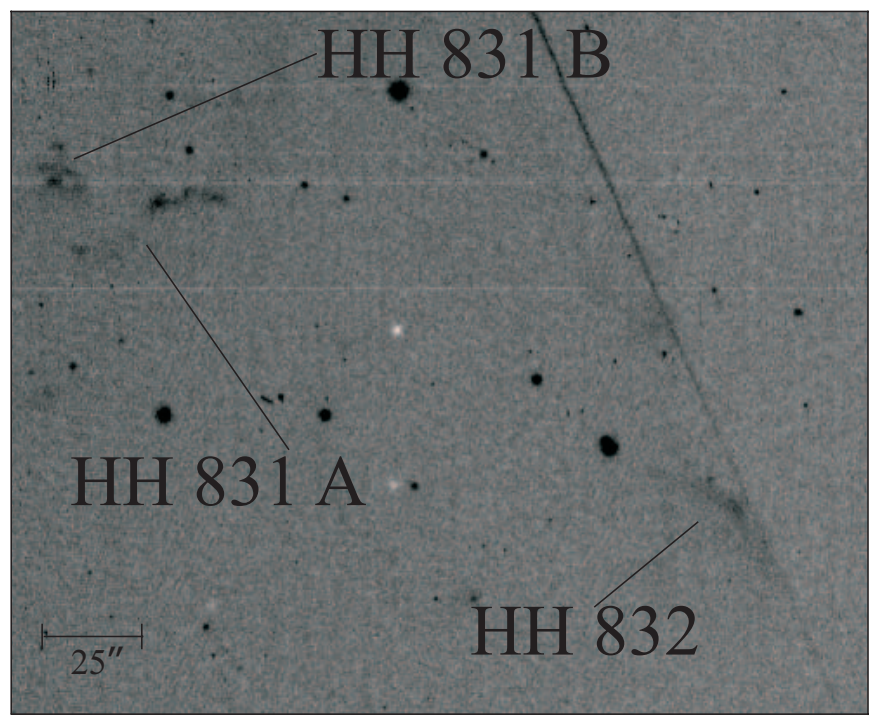

Fig. 8. HH 831 and HH 832 [SII]: [SII] image of the newly discovered objects HH 831 and HH 832. Note that the morphology of HH 831 is quite different here to the $\mathrm{H} \alpha$ image (Fig. 9) and that $\mathrm{HH} 832$ is clearly seen in $[\mathrm{SII}]$ emission.

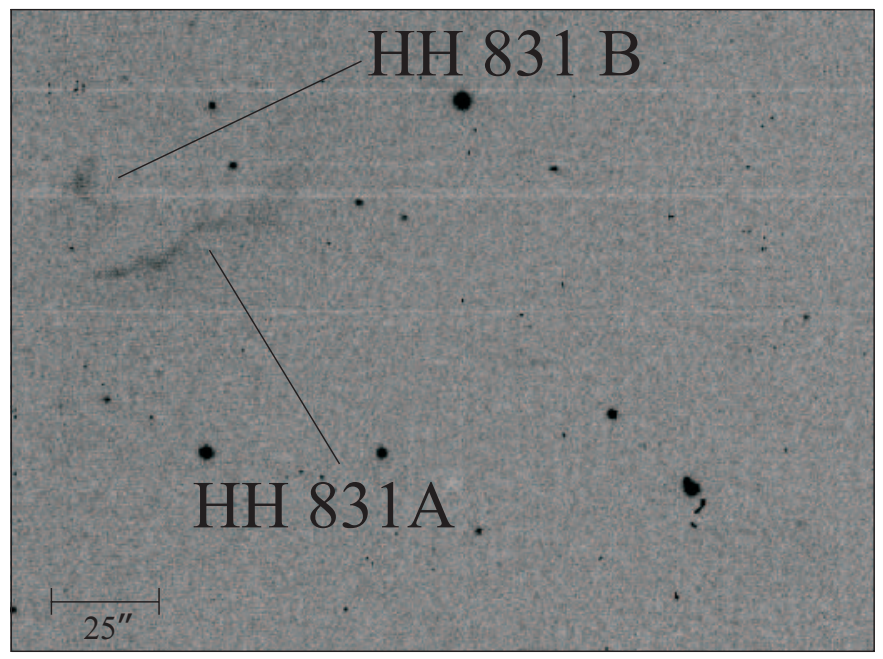

Fig. 9. $\mathrm{HH} 831 \mathrm{H} \alpha$ : $\mathrm{HH} 831$ as seen in $\mathrm{H} \alpha$ emission. Note that this object is much fainter in $\mathrm{H} \alpha$ emission than in [SII] (Fig. 8) and there is no indication of $\mathrm{HH} 832$ here.

CTTSs (Duchêne et al. 1999). Although it was suggested as early as 1986 that RW Aur A itself might be a close binary (Hartmann et al. 1986) it is only in recent years that this has been confirmed with evidence of a low-mass secondary companion, possibly a brown dwarf (Gahm et al. 1999; Petrov et al. 2001). The HH 229 bipolar jet is the only indication of an outflow in the vicinity of RW Aur to date (Hamann 1994; Hirth et al. 1994). Furthermore, long slit spectroscopy confirms RW Aur A as the source of this outflow (Hirth et al. 1994, 1997) as does the 0.' 1 resolution images of Dougados et al. (2000). Recently rotation has been observed at the base of the HH229 jet - one of the first such outflows where rotation has been detected (Coffey et al. 2004).

The blueshifted component of the HH 229 bipolar jet is at a PA of $\sim 130^{\circ}$ with respect to RW Aur A (Dougados et al. 2000;

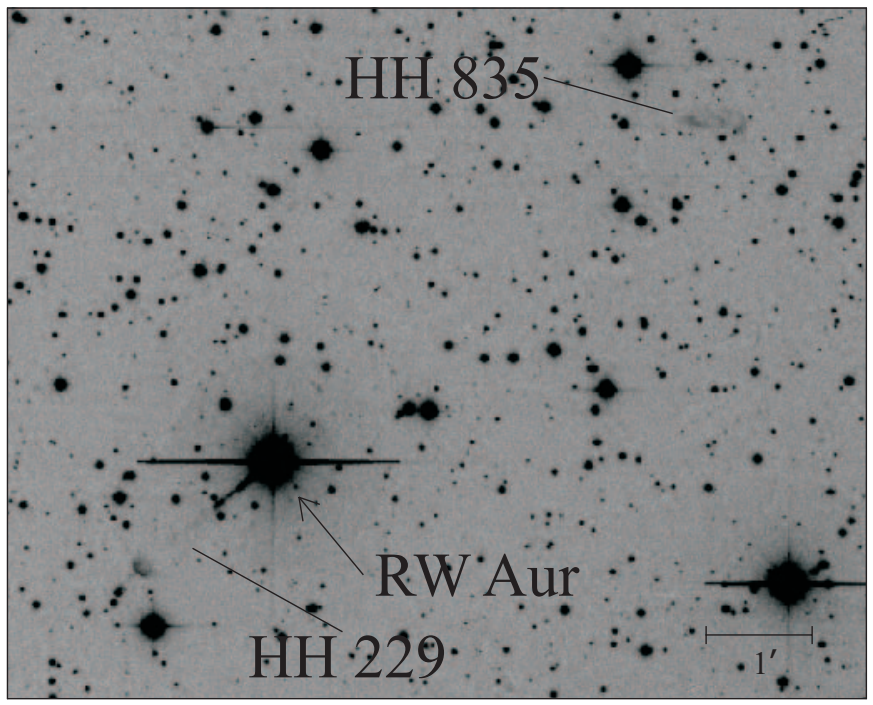

Fig. 10. RW Aur H $\alpha$ : in this image of the RW Aur outflow, the HH 229 jet (and its direction) is clearly seen as is the newly discovered object HH 835, which is seen in more detail in Fig. 11.

Table 5. Angular separations, PAs and spatial extent of the newly discovered HH object in the RW Aur outflow.

\begin{tabular}{llccc}
\hline \hline Object & Source & $\begin{array}{c}\text { Angular } \\
\text { separation }^{a}\end{array}$ & $\begin{array}{c}\mathrm{PA}^{b} \\
{ }^{\circ}\end{array}$ & $\begin{array}{c}\text { Spatial } \\
\text { extent }^{c}\end{array}$ \\
\hline HH 835 & RW Aur & 5.37 & 310 & $30^{\prime \prime} \times 12^{\prime \prime}$ \\
\hline
\end{tabular}

${ }^{a}$ Angular distance from the presumed source.

${ }^{b}$ PA with respect to the presumed source.

${ }^{c}$ Width and length of the object respectively.

Mundt \& Eislöffel 1998; Hirth et al. 1997). [SII] images of Mundt \& Eislöffel (1998) show the redshifted and blueshifted outflows to have lengths of $50^{\prime \prime}$ and $106^{\prime \prime}$ respectively, implying a total projected length of $\sim 0.1 \mathrm{pc}$ at a distance of $140 \mathrm{pc}$. Both sides are well collimated, with a full opening angle for the redshifted jet of less than $6^{\circ}$ (Woitas et al. 2002). The detection of emission in the redshifted jet can be traced back to $0 .^{\prime} 1$ from the source which implies an upper limit of 15 AU for the projected radius of its circumstellar disk.

Our images reveal a large object (HH 835) 5.37 to the northwest of RW Aur (See Fig. 10 and Table 5). We suggest that this object is a bow shock with only the $\sim 30^{\prime \prime}$ long northern wing visible in our $\mathrm{H} \alpha$ images (Fig. 11). Given the position of HH 835 on the known axis of the RW Aur outflow (PA of $310^{\circ}$ with respect to RW Aur) we suggest that this object is part of the redshifted RW Aur A outflow. There is a distance of $\sim 4^{\prime} .6$ between the end of the redshifted jet and HH 835 in which there is no optical evidence for outflow activity. The total observed projected length of the RW Aur outflow from the blueshifted jet to $\mathrm{HH} 835$ is $0.29 \mathrm{pc}\left(7^{\prime}\right)$.

\section{Discussion}

We have shown that five of the seven CTTSs examined in the Taurus-Auriga Cloud (namely CW Tau, DG Tau, DO Tau, HV Tau C and RW Aur) drive outflows of the order of $1 \mathrm{pc}$ 
Table 6. Outflow lengths, dynamical timescales and degree of collimation for the five newly discovered parsec-scale outflows from (Class II) CTTSs and for the serendipitously discovered outflow from the Class I source DG Tau B.

\begin{tabular}{lcccllcl}
\hline \hline Source & $\begin{array}{c}L_{\text {bol }} \\
/ L_{\odot}\end{array}$ & Reference & $\begin{array}{c}\text { Outflow length } \\
/ \mathrm{pc}\end{array}$ & Associated nebula & $\begin{array}{l}\text { Cloud size }^{a} \\
/ \mathrm{pc}\end{array}$ & $\begin{array}{c}\tau_{\text {dyn }} \\
\times 10^{4} \text { yr }\end{array}$ & $\begin{array}{c}\theta_{\text {flow }}{ }^{b} \\
/^{\circ}\end{array}$ \\
\hline DG Tau & $\geq 7.6$ & 1 & 1.09 & LDN 1521 & $7.4 \times 5.4$ & 2.1 & 10.6 \\
DG Tau B & 0.88 & 2 & 0.46 & LDN 1521 & $7.4 \times 5.4$ & 0.9 & 1.8 \\
CW Tau & 2.6 & 3 & 0.98 & LDN 1495 & $3.9 \times 3.2$ & 2.0 & 3.8 \\
HV Tau C $^{c}$ & $\sim 0.5$ & 4 & 0.49 & LDN $1527^{d}$ & $0.03 \times 0.01$ & 1.0 & $4.1^{e}$ \\
DO Tau $^{f}$ & 3.5 & 1 & 0.45 & LDN $1527^{d}$ & $0.03 \times 0.01$ & 0.9 & $3.1^{g}$ \\
RW Aur & $\lesssim 1.1^{\mathrm{h}}$ & 5 & 0.29 & Anonymous & & 0.6 & 4 \\
\hline
\end{tabular}

${ }^{a}$ Visual estimate of the approximate width and length of the associated nebula for each source.

${ }^{b} \theta_{\text {flow }}$ is calculated by taking the width of the most distant shock in both the red - and blueshifted outflows and dividing by the projected distances from the source. The mean value of $\theta_{\text {flow }}$ for both outflows, where both are seen, is given here.

${ }^{c}$ Here we assume that HH 833 and HH 705 are part of the HV Tau C outflow (see Sect. 3.3).

${ }^{d}$ The source is at the edge of this nebula.

${ }^{e}$ This is the opening angle for the blueshifted outflow only - the redshifted jet is too short to measure accurately.

${ }^{f}$ Here we assume that HH 230, HH 831 and HH 832 are part of the DO Tau outflow (see Sect. 3.3).

${ }^{g}$ This is the opening angle for the redshifted outflow only - there is no optically visible blueshifted outflow (see Sect. 3.3).

${ }^{h}$ This is the luminosity of the RW Aur A binary and so is an upper limit for the binary component driving this outflow (see Sect. 3.4). References: 1. Cohen \& Kuhi (1979); 2. Jones \& Cohen (1986); 3. Gomez de Castro (1993); 4. Stapelfeldt et al. (2003); 5. Petrov et al. (2001).

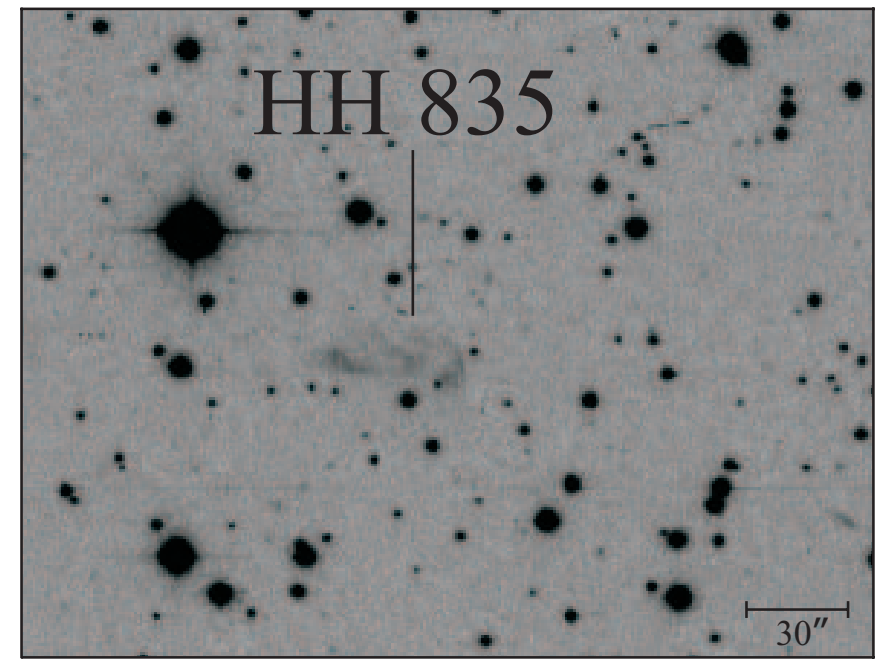

Fig. 11. $\mathrm{HH} 835 \mathrm{H} \alpha$ : $\mathrm{HH} 835$ to the northwest of RW Aur. We suggest that this is the east/west wing of a bow shock, with the north/south wing being much fainter in our optical images.

in length. We have estimated the length, dynamical timescale and degree of collimation for all six of the outflows discussed in Sect. 3 and these are presented in Table 6. Here we examine the parameters and the morphological trends of these CTTS outflows in more detail, and compare them to large-scale outflows from more embedded Class I sources. These trends can be used to infer details about the source, the propagation of the outflow into the ambient medium, and the evolution of the outflows with time.

\subsection{Outflow morphology}

Typically many large-scale outflows from Class I sources are either "S" shaped with the driving source approximately in the middle of the "S" - for example the HH 47 outflow (Heathcote et al. 1996), HH 34 outflow (Bally \& Devine 1994) and the PV Cephei outflow (Reipurth et al. 1997) to name but a few; or the rarer "C" shaped with the source at the apex of the " $C$ " - for example the HH 366 outflow in Barnard 5 (Bally et al. 1996). It can be seen from the images presented in Sect. 3 that many of the outflows from CTTSs show evidence for some variations in PA with time, the effect being most evident in the CW Tau outflow. This outflow delineates an inverted "S" shape centered on CW Tau with the northern part of the "S" more elongated than the southern part. The change in outflow direction is possibly due to precession of the outflow axis (Raga et al. 2001; Masciadri \& Raga 2002).

It has also been noted from large-scale outflows driven by less evolved sources that the size and complexity of the $\mathrm{HH}$ shocks increases with distance from the source. As the supersonic outflow propagates through the parent cloud it interacts with both the ambient medium and slower, previous ejecta producing the $\mathrm{HH}$ shocks observed. Many of these shocks fade quickly with time, it is only the strongest and largest shocks that remain. As a consequence of this, the gap between consecutive $\mathrm{HH}$ objects/complexes increases with distance as the majority of the more distant shocks quickly fade leaving just a few, larger shocks (Reipurth \& Bally 2001; Bally \& Devine 1997). As these extended shocks have undergone many interactions with their surroundings, and have had time to evolve, they will often be complex, chaotic objects rather than the simple knot-like structures seen closer to the young star. Most of the outflows from CTTSs presented here also demonstrate these trends.

\subsection{Outflow length}

From Table 6 we see that the length of the outflows from CW Tau, DG Tau, DO Tau and HV Tau C is of the order 
of $\sim 1 \mathrm{pc}$. This represents an increase over the previously known values by a factor of 480 for the HV Tau C outflow, and factors of between 120 and 160 for the other three. The RW Aur outflow was previously known to be $\sim 2$ ! 7 in length and the revised value is only a factor of $\sim 2.6$ larger $\left(7^{\prime}\right)$. Outflows of the order of 1 pc should readily be expected from Class II low mass stars, the typical age of which is $10^{6}$ years. Even if an outflow has an average velocity of around $50 \mathrm{~km} \mathrm{~s}^{-1}$ (i.e. the minimum velocity required to excite the shocks we see) then over $10^{6}$ years it could extend up to $50 \mathrm{pc}$.

The field of view captured by the Wide Field Camera is $\sim 34^{\prime}$ square which projects to a maximum detectable outflow length of $1.4 \mathrm{pc}$ at the distance of the Taurus-Auriga Cloud. Outflow morphology suggests that the distance between consecutive $\mathrm{HH}$ objects complexes increases exponentially with distance from the source (Sect. 4.1) so if there is emission beyond our field of view then it may easily be on scales of tens of parsecs, near the edges of the Taurus-Auriga Cloud boundaries, and well beyond the field of view of the WFC.

The apparent dynamical timescales for these outflows is estimated in Table 6 , assuming the most distant objects are moving at a velocity of $50 \mathrm{~km} \mathrm{~s}^{-1}$. Values of around $10^{4}$ years are derived thus we are only observing a fraction of the stars' outflow histories.

Our observations suggest that, in all cases, the outflows have blown out of the associated clump. Many of the most distant objects in the outflows are at the clump edges while a few, most notably HH 830 and HH 702 in DG Tau are clearly well beyond. There is little doubt based on statistically estimated lifetimes that these outflows are even larger than our field of view, and thus our data supports the idea that they have blown out of their parent cloud. Large-scale outflows blowing out of their parent cloud is also a well documented occurrence in outflows from Class I low-mass sources, as our own observations of DG Tau B show. We have previously shown that this also occurs for outflows from intermediate-mass YSOs (McGroarty et al. 2004).

\subsection{Degree of collimation}

For the CTTSs outflows discussed here we find a degree of collimation of the order of $\sim 5^{\circ}$ (see Col. 11 of Table 6). This value is in good agreement with the degree of collimation of large-scale outflows from more embedded Class I stars (Mundt et al. 1991). Although our sample of outflows from Class II stars is small, this suggests that collimation remains high even as the source evolves.

\subsection{Have these CTTSs gone through the FU Orionis phase?}

The morphology and location of the $\mathrm{HH}$ objects/complexes associated with the five CTTSs suggest long quiescent outflow phases punctuated by mass ejections of varying strength. According to this scenario, the most violent of the latter have given rise to the most distant (from the YSO), long-lasting and extended $\mathrm{HH}$ complexes that we see today. Reipurth \& Aspin (1997, and references therein) have suggested that these major ejection events occur when the parent star undergoes FU Orionis-type outbursts, or FUor events. Before addressing whether this is the case, and explaining why we think our sample, albeit small, lends considerable weight to the hypothesis, it is worthwhile saying a few words about the FU Orionis phenomenon.

When a YSO undergoes an FUor outburst, its optical brightness increases by several orders of magnitude before decaying back to the pre-outburst luminosity over 50-100 years (Hartmann \& Kenyon 1996). The spectra of these so-called FUors in quiescence is that of a CTTS (Hartmann \& Kenyon 1996), and there are at least nine known FUors (Hartmann \& Kenyon 1996) including the prototypical source - FU Orionis itself (Herbig 1977). Almost immediately after their discovery it was suggested that FUor events could give rise to HerbigHaro outflows (Dopita 1978; Reipurth 1985). More recently, as already stated, Reipurth \& Aspin (1997) investigated this possible connection, basing their conclusions largely on a sample of embedded Class I sources. As it is accepted that in quiescence FUors are CTTSs it seems more appropriate to examine outflows of the latter for any "fossil record" of FU Orionis-type outbursts. In particular it is not obvious a priori that the outburst timescales for Class I and Class II stars are similar.

Examining Table 6 we see that the dynamical timescales of the extended $\mathrm{HH}$ complexes, associated with our CTTSs, are typically $10^{4}$ years. Herbig (1977) and Herbig et al. (2003) have estimated the mean time between successive FUor outbursts is $10^{4}$ years, suggesting a clear link. Moreover the relative number of FUors in comparison to CTTSs also supports this link.

\section{Conclusions}

We have shown here that a number of (Class II) CTTSs, DG Tau, CW Tau, DO Tau, HV Tau C and RW Aur, which were previously known to drive "micro-jets" or short outflows of $\lesssim 1^{\prime}$ ( $\$ 0.04 \mathrm{pc}$ at the distance of the Taurus-Auriga cloud) in length, are actually capable of driving outflows with lengths of the order of $1 \mathrm{pc}$. The serendipitous discovery of a $0.5 \mathrm{pc}$ long outflow from the Class I source DG Tau B is also reported here.

The morphological trends observed in the CTTSs outflows are comparable to those noted in younger sources i.e. increasing distance between successive $\mathrm{HH}$ objects coupled with increased size and complexity with distance from the source. In a few cases, small variations in the direction of propagation of the outflow have been found. The high degree of collimation of the five extended outflows from CTTSs compared well with that observed in the case of extended large-scale outflows from less evolved sources, suggesting that outflows remain focussed even as the source evolves from the Class I to the Class II stage. It is clear that the observed parsec-scale lengths of the CTTS outflows are minimum values and in reality they are much larger. These outflows all show evidence for having blown out of the parent cloud.

The apparent dynamical timescale of these extended outflows is typically a few times $10^{4}$ years. This suggests a linkage between the major accretion events that give rise to the largest $\mathrm{HH}$ complexes and the FU Orionis phenomenon. As FU Orionis stars are CTTSs in quiescence, the extended 
outflows of the latter provide the best "fossil record" to test this linkage.

Acknowledgements. We thank the anonymous referee for helpful comments that clarified the presentation of these results. F.McG. and T.P.R. acknowledge support from Enterprise Ireland. This research has made use of the SIMBAD database, operated at CDS, Strasbourg, France.

\section{References}

Anglada, G., Rodríguez, L. F., Canto, J., Estalella, R., \& Torrelles, J. M. 1992, ApJ, 395, 494

Bacciotti, F., Mundt, R., Ray, T. P., et al. 2000, ApJ, 537, L49

Bacciotti, F., Ray, T. P., Mundt, R., Eislöffel, J., \& Solf, J. 2002, ApJ, 576, 222

Bally, J., \& Devine, D. 1994, ApJ, 428, L65

Bally, J., \& Devine, D. 1997, Herbig-Haro Flows and the Birth of Stars, IAU Symp., 182, 29

Bally, J., Devine, D., \& Alten, V. 1996, ApJ, 473, 921

Beckwith, S. V. W., Sargent, A. I., Chini, R. S., \& Guesten, R. 1990, AJ, 99, 924

Cabrit, S., Edwards, S., Strom, S. E., \& Strom, K. M. 1990, ApJ, 354, 687

Coffey, D., Bacciotti, F., Woitas, J., Ray, T. P., \& Eislöffel, J. 2004, ApJ, accepted

Cohen, M., \& Kuhi, L. V. 1979, ApJS, 41, 743

Devine, D., Bally, J., Reipurth, B., \& Heathcote, S. 1997, AJ, 114, 2095

Dougados, C., Cabrit, S., Lavalley, C., \& Ménard, F. 2000, A\&A, 357, L61

Dopita, A. 1978, A\&A, 63, 237

Duchêne, G., Monin, J.-L., Bouvier, J., \& Ménard, F. 1999, A\&A, 351,954

Edge, A. C., \& Rottgering, H. 1995, MNRAS, 277, 1580

Eislöffel, J., \& Mundt, R. 1997, AJ, 114, 280

Eislöffel, J., \& Mundt, R. 1998, AJ, 115, 1554

Elias, J. H. 1978, ApJ, 224, 857

Gahm, G. F., Petrov, P. P., Duemmler, R., Gameiro, J. F., \& Lago, M. T. V. T. 1999, A\&A, 352, L95

Ghez, A. M., Neugebauer, G., \& Matthews, K. 1993, AJ, 106, 2005

Gomez de Castro, A. I. 1993, ApJ, 412, L43

Hamann, F. 1994, ApJS, 93, 485

Hartigan, P., Edwards, S., \& Ghandour, L. 1995, ApJ, 452, 736

Hartmann, L., Hewett, R., Stahler, S., \& Mathieu, R. D. 1986, ApJ, 309,275
Hartmann, L., \& Kenyon, S. J. 1996, ARA\&A, 34, 207

Heathcote, S., Morse, J. A., Hartigan, P., et al. 1996, AJ, 112, 1141

Herbig, G. H. 1977, ApJ, 217, 693

Herbig, G. H., \& Bell, K. R. 1988, Lick Observatory Bulletin, Santa Cruz: Lick Observatory

Herbig, G. H., Petrov, P. P., \& Duemmler, R. 2003, ApJ, 595, 384

Hirth, G. A., Mundt, R., \& Solf, J. 1997, A\&AS, 126, 437

Hirth, G. A., Mundt, R., Solf, J., \& Ray, T. P. 1994, ApJ, 427, L99

Hirth, G. A., Mundt, R., \& Solf, J. 1994, A\&A, 285, 929

Jones, B. F., \& Cohen, M. 1986, ApJ, 311, L23

Lada, C. J., \& Wilking, B. A. 1984, ApJ, 287, 610

Lada, C. J. 1987, Star Forming Regions, IAU Symp., 115, 1

Lavalley, C., Cabrit, S., Dougados, C., Ferruit, P., \& Bacon, R. 1997, A\&A, 327, 671

Masciadri, E., \& Raga, A. C. 2002, ApJ, 568, 733

McGroarty, F., Ray, T. P., \& Bally, J. 2004, A\&A, 415, 189

Mitchell, G. F., Hasegawa, T. I., Dent, W. R. F., \& Matthews, H. E. 1994, ApJ, 436, L177

Mitchell, G. F., Sargent, A. I., \& Mannings, V. 1997, ApJ, 483, L127

Mundt, R., Brugel, E. W., \& Bührke, T. 1987, ApJ, 319, 275

Mundt, R., \& Fried, J. W. 1983, ApJ, 274, L83

Mundt, R., \& Eislöffel, J. 1998, AJ, 116, 860

Mundt, R., Ray, T. P., \& Raga, A. C. 1991, A\&A, 252, 740

Ogura, K. 1995, ApJ, 450, L23

Padgett, D. L., Brandner, W., Stapelfeldt, K. R., et al. 1999, AJ, 117 , 1490

Petrov, P. P., Gahm, G. F., Gameiro, J. F., et al. 2001, A\&A, 369, 993

Raga, A., Cabrit, S., Dougados, C., \& Lavalley, C. 2001, A\&A, 367, 959

Ray, T. P. 1987 , A\&A, 171, 145

Reipurth, B. 1985, A\&A, 143, 435

Reipurth, B., \& Bally, J. 2001, ARA\&A, 39, 403

Reipurth, B., Bally, J., \& Devine, D. 1997, AJ, 114, 2708

Reipurth, B., \& Aspin, C. 1997, AJ, 114, 2700

Simon, M., Holfeltz, S. T., \& Taff, L. G. 1996, ApJ, 469, 890

Stapelfeldt, K., Burrows, C. J., Krist, J. E., \& WFPC2 Science Team 1997, Herbig-Haro Flows and the Birth of Stars, IAU Symp., 182, 355

Stapelfeldt, K. R., Ménard, F., Watson, A. M., et al. 2003, ApJ, 589, 410

Sun, K., Yang, J., Luo, S., et al. 2003, Chin. J. Astron. Astrophys., 3, 458

Valentijn, E. A. 1981, A\&A, 102, 53

Wichmann, R., Bastian, U., Krautter, J., Jankovics, I., \& Rucinski, S. M. 1998, MNRAS, 301, L39

Woitas, J., \& Leinert, C. 1998, A\&A, 338, 122

Woitas, J., Ray, T. P., Bacciotti, F., Davis, C. J., \& Eislöffel, J. 2002, ApJ, 580, 336 